\title{
Die Begriffe der Äquivalenz, der Gleichheit und der Identität
}

\author{
Ulrich Felgner ${ }^{1}$
}

Online publiziert: 5. März 2020

(C) The Author(s) 2020, korrigierte Publikation 2021

Schlüsselwörter Gleichheit · Identität · Mathematische Logik · Prädikatenkalkül · Strukturalismus

Mathematics Subject Classification (2010) OOA30 • 01A20 • 01A45 - 01A55 • $01 \mathrm{~A} 60 \cdot 03 \mathrm{~A} 05 \cdot 03 \mathrm{~B} 10$

\section{Einleitung}

„Der Begriff der Gleichheit ist uns Mathematikern vielleicht der geläufigste; dennoch läßt sich schwer sagen, was wir unter ihm verstehen." - Mit diesem Satz beginnt GERHARD HESSENBERG sein Buch über die ,Grundlagen der Geometrie ([24], Berlin, 1930, §1). Er kommt nach einer ausführlichen Diskussion zum Schluß, daß die Relation der Gleichheit nichts anderes als eine Äquivalenzrelation sei, also eine symmetrische, transitive und reflexive Relation, und daß auch umgekehrt jede symmetrische, transitive und reflexive Relation eine Gleichheitsbeziehung sei. Die Begriffe Gleichheit und Äquivalenz wären demnach synonym.

Schaut man im ,Mathematischen Wörterbuch“ von J. NAAS \& H.L. SCHMID (Berlin, 1967) nach, so findet man unter dem Stichwort Gleichheit (p. 639) genau dieselbe Definition: „Die Gleichheit ist eine Äquivalenzrelation und umgekehrt kann jede Äquivalenzrelation als eine besondere Art von Gleichheit aufgefaßt werden.“

Aber ist die Gleichheit, die beispielsweise im Urteil $7+5=12$ ausgedrückt wird, nicht mehr als lediglich eine Äquivalenz? Ist sie nicht sogar eine Identität, wie es schon bei Louis Couturat in seinen ,Principes des Mathématiques' (1905 [9], p. 52), bei GotTlob Frege in einem Brief vom 24. 8. 1919 (cf. Frege: ,Wiss.

A correction to this publication is available online at https://doi.org/10.1365/s13291-021-00231-7

\footnotetext{
U. Felgner

felgner@uni-tuebingen.de

1 Mathematisches Institut der Universität Tübingen, Tübingen, Deutschland
} 
Briefwechsel $^{‘}$ [21], pp. 153-156) und auch bei manchen anderen Autoren heißt? $7+5$ und 12 sind doch nur zwei verschiedene Namen ein-und-derselben Zahl. Die mit $7+5$ und 12 benannten Zahlen sind dieselben, d.h. sie sind identisch, und $7+5=12$ ist folglich eine Identität!

Es scheint wohl nicht leicht zu sein, die drei genannten Begriffe, Äquivalenz, Gleichheit und Identität, auseinander zu halten. - Oder handelt es sich vielleicht doch um synonyme Begriffe? - Man kommt sehr schnell in Verlegenheit, wenn man gefragt wird, ob diese Begriffe wirklich synonym sind. - ,Identity is a popular source of philosophical perplexity" heißt es etwas ironisch bei WILLARD VAN ORMAN QUINE ([37], p. 621, bzw. p. 65).

Besondere Probleme scheinen mit dem Begriff der Identität verbunden zu sein. Schon Voltaire hielt sie in seinem ,Dictionnaire Philosophique (Stichwort „Identité") für sehr viel interessanter, als man gemeinhin vermuten möchte: „Ce sujet est bien plus interessant qu'on ne pense“ (VolTaire, Euvres Complètes [40], Band 41, p. 225). LUDWIG WITTGENSTEIN schrieb sogar in einem Brief (vom 29. Oktober 1913) an BERTRAND RUSSELL:

„Die Identität ist der Teufel in Person und ungeheuer wichtig, sehr viel wichtiger, als ich (bisher) glaubte."

(L. Wittgenstein Briefe, Suhrkamp-Verlag Frankfurt/M., 1980, p. 36 \& p. 242. Eine nahezu gleichlautende Formulierung findet sich schon in WITTGENSTEINs Brief vom 17. Oktober 1913 an RuSSELL, p. 34 \& p. 241.)

Die Identität ,fordert das Nachdenken heraus durch Fragen, die sich daran knüpfen und nicht ganz leicht zu beantworten sind" - heißt es bei FREGE in seinem Essay ,Über Sinn und Bedeutung' (1892 [19], p. 26, bzw. p. 143).

FREGE selbst hat diese Herausforderung angenommen und 1879/1893 gezeigt, daß nicht die Gleichheit sondern die Identität ein Kernbegriff der Logik ist. Er hat die Identität (und nicht die Gleichheit) in den von ihm aufgestellten Logik-Kalkül aufgenommen und (in Anlehnung an ARISTOTELES und LEIBNIZ) axiomatisiert. Daß dabei alles, was vom Inhalt der Identität in Sprachen der 1. Stufe ausdrückbar ist, aus dem angegebenen Axiomensystem herleitbar ist, hat 50 Jahre später KURT GÖDEL 1929 in seiner Dissertation an der Universität Wien bewiesen. Die von FREGE angegebene Axiomatisierung des Begriffs der Identität, ist - auf Sprachen der 1. Stufe eingeschränkt - also vollständig.

Wir wollen im Hauptteil unseres Essays die Ergebnisse von ARISTOTELES, LEIBNIZ, FREGE und GÖDEL schildern und erklären. Zuvor wollen wir auf die Geschichte des Auftretens der Begriffe der Gleichheit und der Identität und der mit ihnen verwandten Begriffe der Äquivalenz und der Ununterscheidbarkeit eingehen. Dabei ist bemerkenswert, daß zwar der Begriff der Gleichheit überall in den mathematischen Werken der Antike verwendet wird, der Begriff der Identität hingegen nirgendwo. Der Begriff der Identität wurde erst in der ausgehenden Antike im Umfeld der theologischen Auseinandersetzungen um das Dogma der Trinität geprägt und kommt deshalb auch in den älteren mathematischen Werken nicht vor. In die mathematische Literatur ist das Wort erst in der Neuzeit allmählich eingedrungen.

Wir wollen systematisch vorgehen und in den Abschn. 2-6 zunächst die Unterschiede zwischen den genannten Begriffen aufdecken und sorgfältig erläutern. Während die Gleichheit sich auf sprachlich ausdrückbare Eigenschaften bezieht, ist der 
Begriff der Identität sprachunabhängig. Das macht es so schwierig, den Inhalt des Begriffs der Identität in formalen Sprachen zu fassen. Aus der Unabhängigkeit von der Sprache ergibt sich aber auch, daß der Begriff der Identität (im Gegensatz zum Begriff der Gleichheit) als Grundbegriff der Logik (d.h. als ,logische Konstante“) verwendbar ist, worauf - wie wir bereits oben erwähnt haben - zuerst FREGE hingewiesen hat. Darüber werden wir in den Abschn. 7-9 berichten.

Auf die vielfältigen Probleme, die im Umfeld der Philosophie, der Ontologie und der Sprachphilosophie liegen, werden wir nicht eingehen. (Wir verweisen dazu auf Quine [37], Dilworth [11], Sullivan [39] und die dort zitierte Literatur.) Wir beschränken uns auf die Probleme, die mit den Begriffen der Äquivalenz, der Gleichheit, der Ununterscheidbarkeit und der Identität in der Mathematik und der Mathematischen Logik verbunden sind.

Ich möchte Herrn HORST HISCHER (Universität des Saarlandes) ganz herzlich danken dafür, daß er mich in einem Brief vom 18. 4. 2018 auf einige der oben angegebenen Fragen und Probleme hinwies. Ihm und auch VoLKER PECKHAUS (Universität Paderborn) möchte ich für manche Anregungen, die sie mir während der Abfassung dieses Essays gaben, vielmals danken. Dem Referenten bin ich für einige kritische Bemerkungen zu Dank verpflichtet.

\section{Der umgangssprachliche Begriff der, Gleichheit}

Im ,Deutschen Wörterbuch“ von BROcKHAUS \& WAHRIG (Wiesbaden, 1981) wird der Begriff der Gleichheit, so wie er in der Umgangssprache verwendet wird, sehr treffend wie folgt beschrieben. Es heißt dort, daß zwei Dinge ,gleich“ sind, wenn sie

„,in allen oder den wesentlichen Merkmalen übereinstimmen“.

Zwei verschiedene Dinge können also, auch wenn sie nicht in allen Merkmalen übereinstimmen, dennoch als gleich bezeichnet werden, wenn sie in all den Merkmalen, die im betrachteten Kontext relevant sind, übereinstimmen. Zwei Menschen können beispielsweise unterschiedlich groß sein und auch unterschiedlichen Geschlechts sein, aber nach den Verfassungen vieler Staaten sind sie dennoch ,vor dem Gesetz gleich".

Dieses einfache Beispiel kann den umgangssprachlichen Gebrauch des Gleichheitsbegriffes sehr gut belegen. Es stellt sich aber die Frage, ob dieser Begriff auch in der Mathematik in ähnlicher Weise verwendet wird und schon immer so verwendet wurde. Wie wurde er in früheren Jahrhunderten und wie wurde er in anderen Kulturen verwendet? Um darüber etwas sagen zu können, müssen wir in die etymologischen Wörterbücher schauen und die überlieferten mathematischen Werke studieren.

Den etymologischen Wörterbüchern kann man entnehmen, daß das Wort ,gleich“ vom mittelhochdeutschen Wort ,gelich“ mit der Bedeutung einer mehreren zukommenden Form oder Ge-stalt des Leibes oder des Körpers abgeleitet ist. Dabei steht ,lich“ (auch ,lika“) für „Leib, Körper, Gestalt“ und die Vorsilbe „ge“ deutet eine Kollektiv-Bildung an. Demnach hat ,gleich" die ursprüngliche (umgangssprachliche) Bedeutung von „Übereinstimmung der Gestalt“. Daraus ergab sich im Hochdeutschen die oben angegebene etwas allgemeinere Bedeutung, die eine ,relative Gleichheit“ oder auch eine ,,absolute Gleichheit“" sein kann. 
Im Griechischen hat das Wort isos (iَoऽ) dieselbe Bedeutung: Übereinstimmung

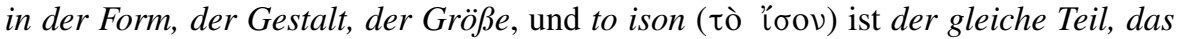
gleiche Verhältnis, aber auch die bürgerliche Gleichheit, das gleiche Recht. Isotês

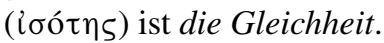

Im Lateinischen steht das Adjektiv aequus für ,gleich“ und aequalitas ist „die Gleichheit" und adaequalitas die Angleichung". ${ }^{1}$ Daraus sind die Wörter aequilibris (waagrecht), aequivalens (gleichwertig) etc. abgeleitet. Die Relation der „Äquivalenz" ist demnach im ursprünglichen Wortsinne eine Relation der Gleichwertigkeit, also eine Relation der Gleichheit, die aber nur die Wertigkeit (oder die Größe) betrifft.

In mathematischen Texten finden sich viele Stellen, die als Belege für den geschilderten Sprachgebrauch dienen können (vergl. dazu aber auch KURT V. FRITZ [22]).

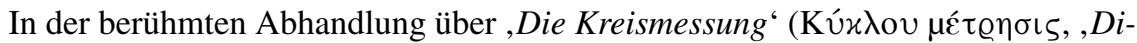
mensio Circuli') von ARCHIMEDES (ca. 280-212 v.u.Z.) heißt es beispielsweise in Satz 1 ([2], Band 1, pp. 231-243):

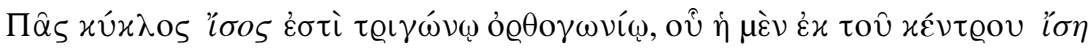

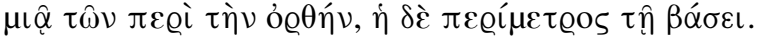

[Jeder Kreis ist einem rechtwinkligen Dreieck gleich, in dem eine der den rechten Winkel einschließenden Seiten gleich dem Radius, die andere gleich dem Umfang des Kreises ist.]

In der Formulierung des Satzes ist der Kreis einem rechtwinkligen Dreieck gleich (!), wobei sich aus dem Zusammenhang ergibt, daß die Gleichheit sich nur auf die Größe der Flächen als wesentliches Merkmal bezieht. Ferner ergibt sich aus dem Zusammenhang, daß die Gleichheit der (geradlinigen) Grundseite des Dreiecks und des (gekrümmten) Umfangs des Kreises sich nur auf die Länge der Linien als wesentliches Merkmal bezieht. Man könnte den Satz von ARCHIMEDES also auch sinngemäß wie folgt übersetzen:

Die Fläche $F(r)$ eines Kreises vom Radius $r$ ist der Größe nach gleich der Fläche des rechtwinkligen Dreiecks, dessen Katheten die Längen $r$ und $U(r)$ haben, wenn $U(r)$ die Größe des Umfangs dieses Kreises ist.

Ebenso werden in den ,Elementen 'EuKLIDs [13] zwei Strecken als gleich angesehen, wenn sie in ihren Längen übereinstimmen. Gleiche Strecken dürfen sich jedoch in ihrer Lage unterscheiden. In der Tat sind für EUKLID Linien nichts anderes als breitenlose Längen (so in der zweiten Definition des ersten Buchs der ,Elemente ${ }^{\text {) }}$. Die wesentlichen Merkmale von Strecken sind demnach, daß sie Länge aber keine Breite haben. Bemerkenswert ist auch das vierte Postulat, das die Gleichheit aller rechten Winkel besagt:

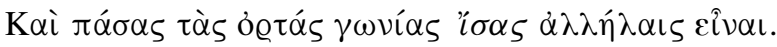

[(Gefordert soll sein), daß alle rechten Winkel untereinander gleich sind.]

Es zeigt sich in diesen Beispielen aus den Werken von ARCHIMEDES und EUKLID, daß von Gleichheit gesprochen werden darf, wenn aus dem Zusammenhang klar ist,

\footnotetext{
${ }^{1}$ Für den Begriff der ,Angleichung“ findet sich eine ausführliche Analyse der Wortbedeutung in dem Essay von U. FELGNER: ,Der Begriff der »Angleichung « $\left(\pi \alpha \varrho \iota \sigma o ́ \tau \eta \zeta\right.$, adaequatio) bei Diophant und Fermat ${ }^{\star}$ [14], 2016.
} 
in welcher Hinsicht eine Übereinstimmung vorliegen soll. Da dieser Zusammenhang aber nicht immer klar ist, hat man sich (einem Vorschlag von GOTTFRIED WILHELM LEIBNIZ folgend $^{2}$ ) im Laufe der Zeit angewöhnt, statt von gleichen Strecken oder gleichen Winkeln etwas genauer von kongruenten (d.h. deckungsgleichen) Strecken bzw. kongruenten Winkeln zu sprechen.

Aber die alte Sprechweise von EUKLID hat sich dennoch sehr lange gehalten. Beispielsweise spricht ADRIEN-MARIE LEGENDRE (1752-1833) in seinen, Éléments de Géométrie، (Paris, 1794) im Satz von der Gleichheit (bzw. Kongruenz) der Scheitelwinkel von zwei sich schneidenden geraden Linien nicht von der Kongruenz, sondern (wie EUKLID, ,Elemente ‘ [13], I.15) von der Gleichheit der Winkel:

„Toutes les fois que deux lignes droites... se coupent, les angles opposés au sommet sont égaux."

[Jedesmal, wenn sich zwei gerade Linien schneiden, sind die gegenüberliegenden Winkel gleich.]

Mit dem Begriff der Gleichheit ist offenbar eine eigenartige Beliebigkeit (oder Ungenauigkeit) verknüpft, die für die Umgangssprache angemessen, für den Gebrauch in der Mathematik aber unpassend ist (vergl. FREGEs Brief an PEANO in FREGE: ,Wiss. Briefwechsel ‘ [21] p. 195). Man hat sich daher in der Neuzeit allmählich angewöhnt, statt von gleichen Linien, etwas genauer von gleichlangen Linien, oder statt von gleichen Körpern von gleichgroßen Körpern etc. zu sprechen. Die Merkmale, worin die Gegenstände übereinstimmen, sollten mitgenannt werden (vergl. FREGEs Brief an LINKE in FreGE: ,Wiss. Briefwechsel ' [21], p. 154, Zeile 3-4)! Der Begriff der Gleichheit ist nur dann klar und exakt, wenn die Gesamtheit aller Merkmale, auf die es ankommt, angegeben wird. Die Relation der Gleichheit ist auch nur dann transitiv, wenn sich die einzelnen Gleichheitsbeziehungen immer auf dieselben Merkmale beziehen.

Wenn nur endlich viele Merkmale in Betracht gezogen werden, dann ist der zugehörige Gleichheitsbegriff in der zugrunde gelegten Sprache definierbar. Wenn jedoch von Gleichheit im absoluten Sinne gesprochen wird, d.h. in Bezug auf alle Merkmale, die in der Sprache, die zum jeweiligen wissenschaftlich betriebenen Fachgebiet gehört, ausdrückbar sind, dann handelt es sich um Ununterscheidbarkeit (lat.: indiscernibilitas, engl.: indistinguishability). Der Begriff der Ununterscheidbarkeit gehört jedoch in der Regel entweder einer infinitären Sprache oder einer Sprache 2. Stufe an. Der Umgang mit diesem Begriff wirft daher besondere Probleme auf. Wir werden darauf in den Abschn. 6-9 zurückkommen.

\section{Das rückverweisende Pronomen ,derselbe 6}

Man sagt, daß $a$ und $b$ identisch sind, wenn die mit $a$ bzw. mit $b$ bezeichneten Dinge ein-und-dieselben Dinge sind, wenn also $a$ und $b$ nur einerlei Ding bezeichnen. Wenn

\footnotetext{
${ }^{2}$ Dieser Vorschlag findet sich in der Beilage eines Briefes vom 8. September 1679 von LEIBNIZ an HUYGENS; siehe ,Leibniz, der Briefwechsel', herausg. Gerhard 1899, pp. 567-575; aber auch ,Euvres de Chr. Huygens', Bd. 8 (1899), pp. 219-224.
} 
$a$ und $b$ identisch sind, dann sind sie auch einander gleich, denn jedes Ding ist sich selbst gleich.

Mit den anaphorischen Pronomina der dritten Person „derselbe“, „dieselbe“ und „dasselbe“ (oder auch ,,der nämliche“ etc.) läßt sich ausdrücken, daß zuvor genannte Dinge identisch sind, also nur ein einziges Ding sind. Im Griechischen steht ,ho

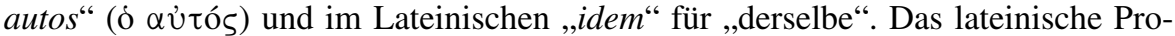
nomen ,idem“ ist durch Zusammenziehung aus dem griechischen ,is“ ( $i$, is) und der Demonstrativ-Endung ,-dem" entstanden (mit langem ,,$i$ " für das ausgefallene „, $s^{\text {") }}$. Dabei sind ,is, ea, $i d^{\prime \prime}$ (,,er, sie, es“; ,der, die, das“; ,dieser, diese, dieses“) die üblichen Pronomina der dritten Person.

Viele gute Beispiele für die Verwendung der Pronomina ,idem“, ,autos“ etc. findet man in zahlreichen mathematischen Werken der Antike und der Neuzeit.

Wenn man beispielsweise die ,Werke ' von ARCHIMEDES ([2], Band 1, p. 8) aufschlägt, dann findet man in der berühmten Abhandlung über ,Kugel und Zylinder

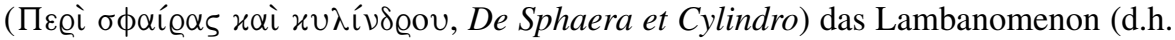
die aus der Erfahrung übernommene Überzeugung):

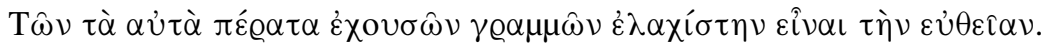

[Von allen Linienstücken, deren Endpunkte dieselben sind, ist die gerade Linie

die kürzeste.]

Hier wird nicht von ,gleichen Endpunkten“, wie manche Übersetzer schreiben (beispielsweise ARTHUR CZWALINA [2], p. 78) sondern von „denselben“ (ta auta) Endpunkten gesprochen, und das ist ein erheblicher Unterschied. Die Endpunkte müssen dieselben sein, sie müssen übereinstimmen, oder mit anderen Worten: sie müssen identisch sein; andernfalls würde das Postulat keinen Sinn machen, da alle Punkte offenbar untereinander gleich sind (vergl. Abschn. 2).

Bemerkenswert ist auch das folgende Axiom: Wenn gleiche Strecken (also kongruente Strecken) dieselben Endpunkte haben, dann sind sie (nach EUKLID, ,Elemente ‘ [13], Buch I, Allg. Grundsatz 9) dieselben, also identisch.

Schließlich erwähnen wir noch, daß in den ,Elementen“ EUKLIDs im ersten Buch der folgende Satz (I.37) bewiesen wird: „Auf derselben Grundlinie zwischen denselben Parallelen gelegene Dreiecke sind einander gleich“. Damit wird dann der etwas allgemeinere Satz (I.38) bewiesen: „Auf gleichen Grundlinien zwischen denselben Parallelen gelegene Dreiecke sind einander gleich“.

Man stellt mit Freude fest, daß EUKLID (im Unterschied zu seinen Vorgängern, vergl. dazu KURT VON FRITZ [22]) überall in seinen ,Elementen', dort wo er Geometrie betreibt, sehr sorgfältig zwischen „,den gleichen“ und „,denselben“ Objekten unterscheidet. In seinen Büchern, die sich mit der Zahlentheorie beschäftigen, wird jedoch immer nur von gleichen Zahlen gesprochen, auch dort, wo wir heute lieber von denselben Zahlen sprechen würden (etwa bei der Bildung von Quadratzahlen in Definition VII.18, etc.). Aber natürliche Zahlen sind ja genau dann (im Hinblick auf ihre Größe) gleich, wenn sie dieselben sind.

Es ist nicht immer leicht zu entscheiden, ob man in gelegentlich auftretenden Situationen von ,gleichen“ oder „,denselben“ Objekten sprechen muß. Als Beispiel wird schon von ARISTOTELES (in seiner ,Topik', I, Kapitel 7, 103a15) diskutiert, ob man das Wasser, das man an verschiedenen Tagen aus einer Quelle schöpft, als dasselbe 
bezeichnen kann. Es ist „,der Art nach dasselbe“, aber nicht „,der Zahl nach dassel-

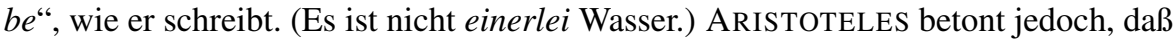
im eigentlichen Sinne nur dann von ,denselben Dingen“ gesprochen werden darf, wenn die dabei verwendeten verschiedenen Namen nur einerlei Ding bezeichnen (103a24-27).

Bemerkenswert ist, daß ARIstoteles im siebten Buch seiner ,Topik', Zeilen 152a31-152b37, auch allgemeine Gesetze für den Umgang mit Aussagen über identische Objekte formuliert, beispielsweise:

(A1): Wenn a und $b$ dasselbe Ding und ebenso a und $c$ dasselbe Ding bezeichnen, dann bezeichnen auch $b$ und $c$ dasselbe Ding.

(A2): Wenn $a$ und $b$ dasselbe Ding bezeichnen, dann mu $\beta$ auch jede Eigenschaft, die dem mit a bezeichneten Ding zugesprochen wird, ebenfalls dem mit b bezeichneten Ding zugesprochen werden.

(A2) handelt nicht nur von den Eigenschaften, die ein Ding per se ( $\left.x \alpha \theta^{\prime} \alpha \dot{\tau} \tau o ́\right)$ besitzt, sondern auch von allen Bestimmungen, die ein Ding bloß akzidentell ( $x \alpha \tau \alpha$ $\sigma \cup \mu \beta \varepsilon \beta \eta x o ́ \varsigma$ ) besitzt. Hervorzuheben ist, daß (A2) nicht gilt, wenn man statt von „denselben“ Dingen nur von ,gleichen“ Dingen spräche - Wir werden in Abschn. 7 darauf zurückkommen und dabei auch die von LEIBNIZ angegebene Variante von (A2) behandeln.

\section{Der Begriff der ,Identität}

Die Geschichte des Aufkommens des Begriffs der „Identität“ ist interessant. Das Wort ,identitas" soll als Übersetzung des griechischen Wortes $\tau \alpha \cup \tau o ́ \tau \eta \varsigma$ (auch

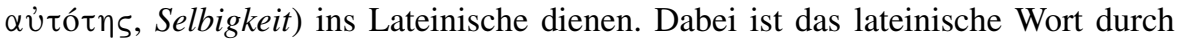
Nominalisierung aus dem Pronomen ,idem“ (derselbe, dieselbe, dasselbe) entstanden. Es ist vermutlich erst in der zweiten Hälfte des 4. Jahrhundert gebildet worden, denn ältere Belege für seine Verwendung sind nicht bekannt. Im ,Thesaurus Linguae Latinae، (Leipzig 1900-1947) wird berichtet, daß die ältesten Belege in den Schriften des Rhetors GAIUS MARIUS Victorinus (das ist ViCTORINUS AFER, ca. 290370, der Lehrer des Hl. Augustinus) gefunden werden können. Er hat es in seinen Schriften gegen die Arianer im Streit um das trinitarische Problem der Theologie häufig verwendet. Es ist also naheliegend anzunehmen, daß es VICTORINUS war, der das lateinische Wort ,identitas“ als Äquivalent für das griechische Wort $\tau \alpha \cup \tau o ́ \tau \eta ऽ$ gebildet hat.

Vom Nomen „Identität“ ist das Adjektiv ,identisch“ abgeleitet. Es bedeutet nicht „,vollkommen übereinstimmend“, wie manche Wörterbücher schreiben, sondern ,,selbig".

Wir bemerken noch, daß VolTAIRE (1694-1778) in seinem ,Philosophischen Lexikon' (1764, Nachdruck in den ,Euvres Complètes' [40], Band 41, pp. 225-229) zum Stichwort „Identite“" schrieb, daß es ,même chose“ bedeuten würde und folglich mit ,mêmeté“ (d.h. Selbigkeit) übersetzt werden könnte:

„Ce terme scientifique ne signifie que même chose. Il pourrait être rendu en français par mêmeté.“ 
[Dieser wissenschaftliche Terminus bedeutet nur »dieselbe Sache«. Er könnte (... ) mit »Selbigkeit « wiedergegeben werden.]

Man spricht also von Identität, wenn ausgedrückt werden soll, daß zwei Bezeichnungen ein-und-dasselbe (also ,einerley“) Ding bezeichnen. Beispielsweise wird der übliche Personalausweis auch Identitäts-Karte (franz.: Carte d'Identité) genannt, da er belegen soll, daß die auf der Karte genannte Person mit der abgebildeten Person übereinstimmt, also einerley Person darstellt.

Ein anderes einfaches Beispiel - es stammt von GoTTLOB FREGE - für den Gebrauch des Begriffs der Identität besagt, daß der Morgenstern mit dem Abendstern identisch ist, da es sich nur um zwei verschiedene Namen ein-und-desselben Gestirns handelt, nämlich der „Venus““ 3 Die Bezeichnungen „Morgenstern“ und ,Abendstern“ haben nicht denselben Sinn, wohl aber dieselbe Bedeutung. - Über „Sinn und Bedeutung“, im Sinne FrEGEs, werden wir in Abschn. 5, Abschn. 7 und Abschn. 8 noch ausführlich sprechen.

Das Wort „Identität“ ist erst in der Neuzeit (um 1700) als Lehnwort ins Deutsche gekommen. Bis dahin war das Wort „Einerleyheit“ üblich, und statt ,identisch“ sagte man ,selbig“, oder ,einerlei“ (vergl. JOHANN HEINRICH LAMBERT [28], p. 91).

In antiken mathematischen Texten taucht weder das griechische Substantiv tautotês noch seine Übersetzung ins Lateinische auf. Statt dessen wird ganz konkret von „ein-und-denselben“ Dingen gesprochen. Eine abstraktere Ausdrucksweise war damals noch nicht nötig.

In der Literatur zur Mathematik und zur Mathematischen Logik taucht das lateinische Wort ,identitas“ erst etwa von 1650 an gelegentlich auf. Von der Mitte des 19. Jahrhunderts an tritt es häufiger auf (z.B. bei Augustus DE MoRGAN [34], 1847, GeORGE Boole [4] 1854, et al.), aber erst vom ausgehenden 19. Jahrhundert an wird es systematisch verwendet. Die Entwicklung gipfelt im Werk von GoTTLOB FREGE 1879/1893 und im Werk von KURT GöDEL 1929. Sie haben erkannt, daß nicht der Begriff der Gleichheit, sondern der Begriff der Identität eine logische Konstante ist, deren Inhalt (auf die Logik der 1. Stufe eingeschränkt) eine vollständige Axiomatisierung besitzt. Wir werden darauf in den Abschn. 8 and 9 zurückkommen.

\section{5 ,Gleichheit ${ }^{`}$ versus , Äquivalenz'}

Nachdem wir die Begriffe der Äquivalenz, der Gleichheit bzw. der Ununterscheidbarkeit und der Identität einzeln besprochen haben, wollen wir sie in den folgenden Abschnitten miteinander vergleichen. Wir beginnen mit einer Gegenüberstellung der Begriffe der Gleichheit und der Äquivalenz. Sind es synonyme Begriffe, so wie es HeSsenberg und NAAS \& Schmid (vergl. Abschn. 1), aber auch PAUl NATORP ([35], p. 128), HERMANN WEYL ([41], p. 9) und andere behauptet haben?

\footnotetext{
${ }^{3}$ DiOgEnes LAËRTIOS hat im 8. Buch, Kap. I, seiner Schrift über, Leben und Meinungen berühmter Philosophen " angemerkt, daß es PYTHAGORAS oder vielleicht auch PARMENIDES war, der als Erster be-

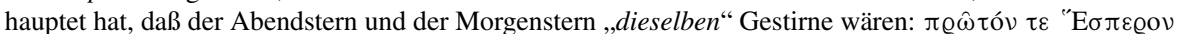

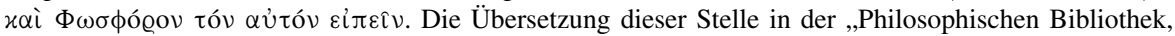
Band 53/54 ([12], Band 2, p. 117) mit „Identität des Abendsternes und des Morgensternes“ ist sinngemäß richtig, aber philologisch nicht ganz korrekt.
} 
Zum Begriff der Äquivalenzrelation wollen wir noch erwähnen, daß er als terminus technicus der Mathematik zuerst von GIUSEPPE PEANO 1894 eingeführt wurde. In seiner Schrift ,Notations de Logique Mathématique [36], p. 168, nannte er eine 2-stellige Relation eine „Äquivalenzrelation“, wenn sie (genauso wie die Gleichheitsbeziehung) im Sinne von Augustus De Morgan (,Syllabus of a System of Logic “, London 1860) reflexiv, symmetrisch und transitiv ist. PEANO behauptete nicht, daß die Begriffe der Gleichheit und der Äquivalenz synonym wären. Diese Behauptung haben erst Natorp, Weyl, Hessenberg, NAAS \& Schmid und andere aufgestellt. Sie ist richtig und läßt sich wie folgt beweisen.

(1) Wir zeigen, daß jede Äquivalenz-Relation , , “ auf einer Menge $M$ mit einer auf $M$ erklärten Gleichheits-Relation übereinstimmt. Wir werden dazu die Elemente von $M$ nur im Hinblick auf das Merkmal, in welcher Äquivalenzklasse von , 〜“ sie liegen, vergleichen. Dann sind zwei Elemente von $M$ genau dann ,gleich“, wenn sie in derselben Äquivalenzklasse liegen. Die vorgegebene ÄquivalenzRelation , “ "fällt also mit der so definierten Gleichheitsrelation zusammen.

(2) Es ist klar, daß auch umgekehrt jede Gleichheitsrelation auf einer Menge $M$ mit einer Äquivalenzrelation auf $M$ übereinstimmt. Die Gleichheitsrelation ist ja selber eine Äquivalenzrelation.

Aus (1) und (2) ergibt sich, daß jede Gleichheitsrelation eine Äquivalenzrelation und umgekehrt auch jede Äquivalenzrelation eine Gleichheitsrelation ist. Die Begriffe der Äquivalenz und der Gleichheit haben also dieselbe Bedeutung, sie sind synonym $^{4}$ ( $\sigma \cup \nu \omega ́ \nu \cup \mu \varsigma_{S}=$ dasselbe benennend). Sie haben also dieselbe Extension, aber sie haben nicht dieselbe Intension. Niemand würde in einem mathematischen Kontext den Ausdruck ,ist gleich" durch ,ist äquivalent" ersetzen - auch nicht umgekehrt. Anders als im Begriff der Gleichheit muß im Begriff der Äquivalenz nicht explizit auf die Übereinstimmung der jeweils relevanten Merkmale Bezug genommen werden.

\section{6 ,Ununterscheidbarkeit‘ versus ,Identität ${ }^{6}$}

Ununterscheidbarkeit ist eine starke Form von Gleichheit, die man auch als absolute Gleichheit oder Gleichheit in jeder Hinsicht bezeichnen kann. Dieser Begriff wurde schon in der Stoa betrachtet, wie aus einer Bemerkung hervorgeht, die PLUTARCH

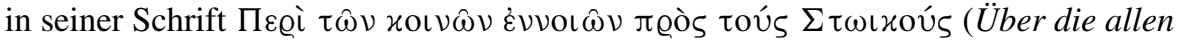
gemeinensamen Begriffe - wider die Stoiker) gemacht hat (dort Kapitel XLIV, über das Individuations-Prinzip; Wir werden darüber in Abschn. 7, Fußnote 7, sprechen).

Wir wollen im Folgenden zeigen, daß (jedenfalls in der heute üblichen strukturalistisch begründeten Mathematik) die Begriffe der Ununterscheidbarkeit und der Identität nicht dasselbe bedeuten. Daraus folgt dann sofort, daß auch die Begriffe der Gleichheit und der Identität nicht bedeutungsgleich sind.

\footnotetext{
${ }^{4}$ Die heute verfügbaren Wörterbücher der deutschen Sprache geben für das Lehnwort ,synonym“ zumeist die folgenden Erklärungen: „,bedeutungsgleich, inhaltlich übereinstimmend, sinnverwandt“. Hier

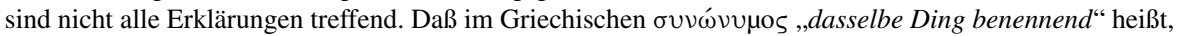
geht aus einem alten, hochgeschätzten Kommentar von ADRIAN TURNEBUS (Paris, 1556) zu einer Stelle in QUINTILIANs ,Institutio Oratoria', Buch VIII, Kap. 3, hervor.
} 
Sei dazu $\mathscr{L}$ irgendeine (formale) Sprache (der ersten Stufe), so wie sie in der Mathematischen Logik und Modelltheorie heute üblicherweise betrachtet wird (vergl. CHANG-KeISLER, ,Model Theory“ [7], p. 18ff). Sei $\mathfrak{M}=\langle M, \ldots\rangle$ eine $\mathscr{L}$-Struktur, d.h. eine nicht-leere Menge $M$, auf der gewisse Funktionen, Relationen und Elemente ausgezeichnet sind, die erlauben, jedes in $\mathscr{L}$ auftretende (primitive) Zeichen zu interpretieren. Dann sind zwei Elemente $a$ und $b$ von $M$ definitionsgemäß in $\mathfrak{M}$ ununterscheidbar, wenn sie dieselben in $\mathscr{L}$ ausdrückbaren Eigenschaften (mit einer einzigen freien Variablen) besitzen.

In der Modelltheorie kann man (unter Verwendung des Kompaktheits-Satzes von KuRT GöDEL (1931) und ANATOL I. MALZEW (1936) und des kombinatorischen Satzes von FRANK P. RAMSEY (1930)) den folgenden Satz beweisen (vergl. CHANG-KeISLER: ,Model Theory‘ [7], p. 148):

Satz 1 Jede in einer (formalen) Sprache $\mathscr{L}$ (der 1. Stufe) formulierte Theorie T, die wenigstens ein unendliches Modell besitzt, besitzt auch ein Modell, in dem es eine unendliche Menge von paarweise ununterscheidbaren Elementen gibt.

„Ununterscheidbarkeit“ und „Identität“ bedeuten also nicht dasselbe. Der angegebene Satz 1 gilt, wie der Beweis zeigt, jedoch nur in einer Mathematik, die auf mengentheoretischem Fundament vom formalen Standpunkt aus betrieben wird (vergl. dazu U. Felgner ,Philosophie der Math` [15], Kapitel 18). Wenn ein anderer Standpunkt eingenommen wird, dann ist es fraglich, ob die zum Beweis von Satz 1 benötigten Konstruktionen von Strukturen und Modellen noch zur Verfügung stehen. Es könnte sein, daß sie nicht zur Verfügung stehen und der obige Beweis nicht mehr durchgeführt werden kann. Beispielsweise vertrat GOTTFRIED WILHELM LEIBNIZ (1.7.1646-14.11.1716) den Standpunkt, ,daß man unter den sinnlich wahrnehmbaren Dingen niemals zwei ununterscheidbare findet, und daß man z.B. in einem Garten keine zwei Blätter... finden wird, die einander vollkommen gleichen" (LEIBNIZ im 5. Brief vom 18. 8. 1716 an SAMUEL CLARKE), woraus sich ergibt, daß in einer Mathematik, in der nur die sinnlich wahrnehmbaren Gegenstände betrachtet werden und keine Abstraktionen oder Idealisierungen vorgenommen werden, Ununterscheidbarkeit und Identität dasselbe bedeuten. Die Frage, ob Identität und Ununterscheidbarkeit dasselbe bedeuten, kann also von der Mathematik allein mit ihren Methoden nicht beantwortet werden, da ihre Beantwortung von grundsätzlichen philosophischen Vorentscheidungen über den ontologischen und den epistemologischen Status der Mathematik abhängen.

Unabhängig vom eingenommenen Standpunkt können wir aber festhalten, daß die Begriffe der „Ununterscheidbarkeit“ und der „Identität" verschiedenen Kategorien angehören. Der Begriff der Identität macht eine ontologische Aussage - nämlich die Einerleiheit von Dingen -, während im Begriff der Gleichheit - genauso wie im Begriff der Ununterscheidbarkeit - nur etwas über die sprachlich ausdrückbaren Eigenschaften von Dingen ausgesagt wird. Der Begriff der Identität ist zudem sprachunabhängig, während der Begriff der Gleichheit, wie wir in Abschn. 2 ausgeführt haben, ganz wesentlich von der zugrundegelegten Sprache abhängig ist. 


\section{Die Definitionen von LEIBNIZ}

BLAISE PASCAL (1623-1662) hat in einem unvollendeten, posthum veröffentlichten Essay, der ,De L'Esprit Géométrique ' genannt wird, behauptet, daß sich der Begriff der Gleichheit nicht definieren lasse. ${ }^{5}$ Er hielt diesen Begriff für einen ,primitiven“ Begriff, der allen Menschen, die der Sprache mächtig sind, unmittelbar vertraut sei und insofern auch nicht definiert werden müsse. Die Art dieses Vertrautseins hielt er für ein natürliches Prinzipienwissen des Herzens, ein ,sentiment du coeur“, also ein Wissen, das jede Rationalität übersteigt.

LEIBNIZ war ganz anderer Ansicht. Er war von der Definierbarkeit überzeugt und hat in verschiedenen Schriften sowohl für den Begriff der Gleichheit als auch für den Begriff der Identität Definitionen vorgeschlagen. Er teilte mit den Rationalisten seiner Zeit die Ansicht, daß sich alle mathematischen Wahrheiten allein aus den Inhalten, die in den Definitionen der jeweils relevanten Grundbegriffe niedergelegt sind, beweisen lassen - und deshalb legte er auf Definitionen so großen Wert!

(I) Eine Definition der Gleichheit gab LEIBNIZ (in Anlehnung an ARISTOTELES, vergl. Abschn. 3) in seiner Schrift: ,Prima calculi magnitudinum elementa demonstrata in additione et subtractione, usuque pro ipsis signorum + et - " (posthum publiziert in ,Math. Schriften“ [29], Bd. 7, pp. 77-82, vergl. auch LEIBNIZens ,Dialog zur Einführung in die Arithmetik u. Algebra', herausgegeben von E. KNOBLOCH, 1976 [31], p. 33):

„Aequalia sunt quorum unum alteri substitui potest salva magnitudine. Et ita designatur $a=b . "$

[Zwei Größen $a$ und $b$ sind gleich, wenn die eine die andere ersetzen kann, unter Bewahrung der Größe. Und dies wird mit $a=b$ bezeichnet.]

LEIBNIZ bewies in der angegebenen Schrift, daß die so definierte Relation reflexiv, symmetrisch und transitiv ist (ohne diese Bezeichnungen zu benutzen). Sie ist also eine Äquivalenzrelation und folglich auch eine Gleichheits-Relation im hergebrachten Sinne. Er bewies auch das Axiom:

„Si aequalibus addas aequalia, fiunt aequalia.“

[Wenn Gleichem Gleiches hinzugefügt wird, entsteht Gleiches],

$a=b \quad \& \quad x=y \quad \Rightarrow \quad a+x=b+y$.

Das ist das zweite allgemeine Axiom im ersten Buch der, Elemente EUKLIDS.

(II) Eine Definition des Begriffes der Identität gab LEIBNIZ in seiner Schrift: ,Non inelegans specimen demonstrandi in abstractis' (etwa aus den Jahren 16851687, cf. LEIBNIZ ,Schriften zur Logik. . ‘ [32], 1992, pp. 153-177). In einem Essay

\footnotetext{
${ }^{5}$ Wenn man nur fragt, ob in einer einzelnen mathematischen Theorie der Begriff der Gleichheit definierbar ist, dann hängt die Antwort von der Ausdruckskraft der zugrunde gelegten Sprache, der Reichhaltigkeit der vorausgesetzten Axiome und der Anzahl der für die Gleichheit relevanten Merkmale ab (vergl. Abschn. 2). Im Falle der ZERMELO-FRAENKEL-SKOLEM'schen Mengenlehre - beispielsweise - kann man, wenn man den Begriff der Gleichheit nicht zur Logik zählen will, die Gleichheit auch durch die folgende Definition einführen: $x=y \Leftrightarrow \forall z(z \in x \Leftrightarrow z \in y) \& \forall u(x \in u \Leftrightarrow y \in u)$, vergl. FRAENKEL \& BAR-HILLEL \& LEVY, 1973 [16], pp. 26-27, ,attitude (c)“.
} 
zum Logik-Kalkül heiß es (cf. L. Couturat: ,Opuscules et Fragments inédits de Leibniz' [8], p. 259) fast wortgleich:

„Eadem sunt, quorum unum alteri substitui potest salva veritate. signum autem est $\infty$, ut $A \infty B$.“

[Identisch sind solche Dinge, von denen das eine das andere ersetzen kann, ohne daß sich die Wahrheit ändert. Das Zeichen dazu ist $\infty$, also $A \infty B$.]

Die Definition könnte etwas ausführlicher wie folgt ausgesprochen werden: Identisch sind die Dinge a und $b$ genau dann, wenn jede Aussage $\Phi$, in der a genannt wird, wahr bleibt, sobald man a durch b ersetzt, und umgekehrt auch jede Aussage $\Psi$, in der $b$ genannt wird, wahr bleibt, sobald man $b$ durch a ersetzt. Diese Definition bezieht sich auf alle Aussagen, die in einer Sprache $\mathscr{L}$ aufgeschrieben werden können. (Sie hängt daher von der zugrunde gelegten Sprache $\mathscr{L}$ ab.) Um Widersprüche zu vermeiden, darf $\mathscr{L}$ keinen Selbstbezug zulassen (vergl. IGNACIO ANGELELLI [1]).

Auch diese von LEIBNIZ gegebene Definition lehnt sich offenbar an das von ARISTOTELES formulierte Gesetz (A2) an, siehe Abschn. 3. Aber für ARISTOTELES ist die Ersetzbarkeit ,salva veritate“ nur eine notwendige Bedingung für Identität, während sie für LEIBNIZ eine notwendige und hinreichende Bedingung ist.

Die Identität der Objekte $a$ und $b$ hat LEIBNIZ mit $a \infty b$ bezeichnet (in Anlehnung an das von DESCARTES verwendete Zeichen $\alpha$, stilisiert: $\alpha$, für ,,aequalitas“). Wir ziehen es vor (wie heute üblich), die Identität der Objekte $a$ und $b$ mit $a \equiv b$ zu bezeichnen. Dann können wir die LEIBNIZ'sche Definition der Identität wie folgt formalisieren und zugleich etwas genauer formulieren:

$$
\forall x \forall y\left(x \equiv y \Leftrightarrow \forall \Phi\left(\Phi \Leftrightarrow \Phi^{*}\right)\right)
$$

wobei $\Phi$ die Menge aller Formeln einer vorgegebenen Sprache $\mathscr{L}$ durchläuft, in denen die Variable $x$ frei vorkommt und $y$ eine Variable ist, durch die $x$ in $\Phi$ frei ersetzt werden kann, und $\Phi^{*}$ aus $\Phi$ dadurch hervorgeht, daß an einigen Stellen, an denen $x$ frei vorkommt, $x$ durch $y$ ersetzt wird. ${ }^{6}$

In dieser Definition (\#) wird, genau genommen, nur der Begriff der Ununterscheidbarkeit definiert. Da aber LEIBNIZ von der Gültigkeit des Prinzips der Individuation überzeugt war, und sich aus diesem Prinzip ergibt, daß Ununterscheidbarkeit und Identität dasselbe besagen, kann man nachvollziehen, warum LEIBNIZ von der Korrektheit seiner Definition überzeugt war. ${ }^{7}$

\footnotetext{
${ }^{6}$ Sei $\Phi$ eine Formel, $x$ eine Variable und sei $t$ ein Term. Dann ist $x$ in $\Phi$ durch $t$ frei ersetzbar, wenn an jeder Stelle, an der $x$ in $\Phi$ frei vorkommt, keine Variable, die in $t$ vorkommt, in den Wirkungsbereich eines Quantors gerät.

${ }^{7}$ Bereits in der Antike wurde gelegentlich die Ansicht vertreten (vergl. Abschn. 6), daß es in der natürlichen Umwelt keine zwei Dinge gibt, die in allen Merkmalen übereinstimmen. Jedes Ding wäre also ein Individuum in dem Sinne, daß es sich von jedem anderen Ding in wenigstens einer Eigenschaft unterscheidet. Das Prinzip der Individuation (principium individuationis) besagt, daß jedes Ding individualisierbar ist, unter allen übrigen Dingen sich als ein Individuum erweist. Wenn die Gültigkeit des (umstrittenen) Prinzips der Individuation angenommen wird, dann wären ununterscheidbare Dinge (d.h. absolut gleiche Dinge) offenbar identisch. LEIBNIZ behandelte das Prinzip der Individuation in seiner, Dissertatio Metaphysica de Principio Individui ' und in seinen ,Nouveaux Essays sur l'entedement humain“ ([30], II, Kap. 27). Für eine sorgfältige Untersuchung dieses Prinzips verweisen wir auf R. KAUPPI [27].
} 
Die LEIBNIZ'schen Definitionen haben dennoch eine große Wirkung gehabt. HERMANN GRASSMANN (1809-1877) beispielsweise verwandte sie in seinem berühmten ,Lehrbuch der Arithmetik، (Berlin 1861, p. 1) und ebenso RICHARD DEDEKIND in seinem Essay ,Was sind und was sollen die Zahlen?' (1888 [10], §1), sowie GotTlob FREGE in seinen ,Grundlagen der Arithmetik“ (Breslau 1884, p. 76) und DAVID HILBERT \& WILHELM ACKERMANN in ihrem Werk, Grundzüge der theoretischen Logik' (1928, [26], p. 83).

Auch Alfred North Whitehead (1861-1947) und Bertrand Russell (1872-1970) haben die LEIBNIZ'sche Definition der Identität im ersten Band ihrer ,Principia Mathematica“ (Cambridge 1910 [42], Def. 13.01) übernommen, sind aber dafür sehr heftig kritisiert worden - insbesondere von FRANK RAMSEY [38] und Ludwig WitTgenstein [43]. Das Prinzip der Individuation ist in der heute üblichen strukturalistisch aufgebauten Mathematik auf mengentheoretischer Grundlage nicht gültig - wie wir in Abschn. 6 gesehen haben - und daher ist die LEIBNIZ'sche Definition als Definition der Identität in dieser Mathematik nicht verwendbar.

WitTGENSTEIn kritisierte in seinem ,Tractatus Logico-Philosophicus ' (1918) in 5.5301, daß die Identität zwar etwas sehr Banales wäre, daß aber dennoch jeder Versuch, den Begriff der Identität zu definieren, zirkelhaft sein müsse (vergl. dazu auch WitTGEnsteins , Vorlesungen 1930-1935‘, [43], p. 338). Hinzukommt, daß sowohl die LEIBNIZ'sche Definition der Gleichheit als auch die der Identität nicht der Logik der ersten Stufe angehören, sondern einer Logik der zweiten Stufe, da über alle Eigenschaften quantifiziert wird.

Wir müssen festhalten, daß aus den genannten Gründen die von LEIBNIZ vorgeschlagenen Definitionen als Definitionen der Gleichheit bzw. der Identität beim Aufbau mathematischer Theorien nicht übernommen werden können. Sie könnten nur dort übernommen werden, wo das Prinzip der Individuation gültig ist. Überdies scheint auch eine korrekte, explizite Definition des Begriffs der Identität in Sprachen der 1. Stufe vermutlich gar nicht möglich zu sein.

Aber es gibt einen Ausweg, auf den zuerst GotTlob Frege $(1879,1893$ [17, 20]) und danach auch DAVID HILBERT (1904 [25]) hingewiesen haben. Darüber wollen wir in den folgenden Abschn. 8 \& 9 sprechen.

\section{Der Begriff der ,Identität ${ }^{6}$ in der Mathematischen Logik (GoTTLOB FREGE)}

Das Problem, ob der Begriff der Gleichheit - oder eventuell eine Abschwächung oder eine Verstärkung dieses Begriffs - sich an die Seite der klassischen Grundbegriffe der Logik ,nicht, und, oder, impliziert, für alle, es gibt,... “ etc. stellen läßt, hat wohl zuerst FREGE bearbeitet. Einen ersten Lösungsversuch hat er 1879 in seiner ,Begriffsschrift ‘ [17] veröffentlicht. Er meinte, daß der Begriff der ,Inhaltsgleichheit “ der geeignete Begriff sei.

\footnotetext{
Die entgegengesetzte Ansicht, daß ununterscheidbare Dinge der realen Umwelt keineswegs identisch sein müssen, hat G.E. MoORE (1873-1958) in einem Aufsatz verteidigt, aber natürlich nicht durch Beweise erhärten können, cf. MOORE [33].
} 
Was unter dem „Inhalt“ eines Begriffes (,compréhension de l'idée“) zu verstehen ist, hatte Antoine ARnAuld (1612-1694) in seinem Buch ,La Logique, ou L'Art de Penser' (auch ,Logique de Port-Royal' genannt, Paris 1662, [3]) beschrieben. Er schrieb dort im ersten Teil, Kap. VI, p. 31:

„J'appelle compréhension de l'idée, les attributs qu'elle enferme en soi, et qu'on ne peut ôter sans la détruire."

[Als Inhalt einer Idee bezeichne ich die (Gesamtheit der) Attribute, die die Idee in sich einschließt, und die man nicht wegnehmen kann, ohne sie (die Idee) zu zerstören.]

Diese Fassung des Inhaltsbegriffes wurde in der Folgezeit von nahezu allen Autoren in ihren Monographien zur Logik übernommen (etwa KANT/JÄSCHE 1800, JOHANN FRIEDRICH FRIES 1819 (p. 107ff), FRIEDRICH EDUARD BENEKE 1842 (Bd. 1, p. 84ff), Adolf Trendelenburg 1862 (Bd. 2, p. 222ff), et al.). Zwei Begriffe (oder zwei Ideen) haben demnach ,denselben Inhalt“", wenn sie dieselben Attribute ${ }^{8}$ in sich schließen.

FREGE hat diese Fassung des Inhaltsbegriffes weitgehend übernommen. Für ihn ist der „Inhalt“ eines Namens die Gesamtheit aller Eigenschaften, die der mit diesem Namen bezeichnete Gegenstand wesentlich (!) besitzt. Ein Name, unter den kein uns vorgegebener Gegenstand fällt, hat nach FREGE keinen Inhalt (vergl. FREGEs Polemik gegen HANKEL in seinen ,Grundlagen der Arithmetik' [18], pp. 105-107). FREGE geht davon aus, daß die Mathematik über einen Gegenstandsbereich verfügt, der ihr vorgegeben ist und aller Formalisierung vorangeht. Er vertritt den ,inhaltlichen Standpunkt" und lehnt den ,formalen Standpunkt" ab, der davon ausgeht, daß die mathematischen Gegenstände nur als Elemente von geeigneten Strukturen existieren und die geforderten Eigenschaften nicht wesenhaft (per se), sondern nur per accidens im Sinne dieser Strukturen haben (vergl. Abschn. 3).

In seiner ,Begriffsschrift ${ }^{\star}$ aus dem Jahre 1879 führt FREGE einen sehr starken Gleichheitsbegriff ein, den er ,Inhaltsgleichheit“" nennt. Zwei Namen $A$ und $B$ sind inhaltsgleich, in Zeichen $A \equiv B$, wenn die von ihnen bezeichneten Gegenstände dieselben Attribute (!) besitzen (FREGE: ,Begriffsschrift ${ }^{\star}$, p. 15). In den Logik-Kalkül kann er von dieser Definition nur das aufnehmen, was formalisierbar ist. Es sind dies (in Anlehnung an ARISTOTELES und LEIBNIZ) die folgenden „Gleichheits-Axiome“ ([17], p. 50) - wobei wir die heute übliche Notation verwenden ${ }^{9}$ :

(i) $\forall x(x \equiv x)$,

\footnotetext{
${ }^{8}$ Attribute sind die wesentlichen Eigenschaften einer Idee (oder eines Objektes) im Unterschied zu den Akzidenzien, den unwesentlichen, nur (aufgrund von Zufälligkeiten, oder von Postulaten etc.) zugeschriebenen Eigenschaften (ARISTOTELES, ,Topik‘. Bd. 1, Kap. 5, 102b4).

${ }^{9}$ FREGE benutzt in (i) \& (ii) nicht, wie heute üblich, Variablen, die mit jedem Element einer vorgegebenen Struktur belegt werden können, sondern ,unbestimmt andeutende“ lateinische Buchstaben - wie er sagt -, d.h. lateinische Buchstaben $A, B, \ldots$, die man nur durch Namen ersetzen darf, die einen Gegenstand bedeuten.

FREGE entwickelte die Logik vom inhaltlichen Standpunkt aus. Sie wurde in den ersten Jahrzehnten des 20. Jahrhunderts vom formalen Standpunkt aus in einen Aufbau auf der Grundlage des Strukturalismus' unter Verwendung der axiomatischen Mengenlehre transformiert. Dabei erhielt sie ihr heute übliches Erscheinungsbild. Von diesem geänderten Standpunkt aus ist die FREGE'sche Denkweise nicht immer nachvollziehbar und wird daher auch in der Literatur oft mißverständlich dargestellt.
} 
(ii) für jede $\mathscr{L}$-Formel $\Phi$ die Aussage: $\forall x \forall y\left(x \equiv y \Rightarrow\left(\Phi \Rightarrow \Phi^{*}\right)\right)$.

Hier in (ii) ist $\mathscr{L}$ die Sprache der betrachteten mathematischen Disziplin ${ }^{10}$ und $\Phi^{*}$ entsteht aus $\Phi$ wie in Abschn. 7, (\#) angegeben.

Wir bemerken zunächst, daß aus den beiden Gleichheits-Axiomen (i) \& (ii) sehr leicht auch die Transitivität und die Symmetrie der Relation „„“ gefolgert werden kann. Also ist „,“ eine Äquivalenz-Relation. Aber die Relation ,„”“ ist von der zugrundegelegten Sprache $\mathscr{L}$ abhängig, denn zwei Namen, die in Bezug auf die Ausdrucksfähigkeit von $\mathscr{L}$ inhaltsgleich sind, können in Bezug auf eine Expansion von $\mathscr{L}$ ungleiche Inhalte haben. Das hat FREGE nicht bedacht. Der Begriff der „Inhaltsgleichheit“ verletzt das „Prinzip der Identität“ der klassischen Logik (zumeist in der Form $\mathfrak{U}=\mathfrak{U}$ mitgeteilt) von Begriffen bei Spracherweiterungen und kann daher keinen Platz in der Logik beanspruchen.

Im Jahre 1888 erschien RICHARD DEDEKINDs Monographie ,Was sind und was sollen die Zahlen?', in der DEDEKIND gleich im ersten Abschnitt deutlich macht, daß er die Gleichheit von Zahlen als Identität auffaßt. Er schreibt:

„Ein Ding ist vollständig bestimmt durch alles das, was von ihm ausgesagt oder gedacht werden kann. Ein Ding a ist dasselbe wie b (identisch mit b), und $b$ dasselbe wie $a$, wenn alles, was von a gedacht werden kann, auch von $b$, und alles, was von bilt, auch von a gedacht werden kann."

DEDEKIND behauptet hier (ohne Begründung) zunächst die Gültigkeit des Prinzips der Individuation (siehe Fußnote 7 in Abschn. 7) und folgert daraus die Gültigkeit einer Identitäts-Aussage, die sich der überlieferten Definition der Identität (ARISTOTELES, LEIBNIZ) anlehnt.

DEDEKINDS Hinweis auf die Identität mag FREGE sehr zu Denken gegeben haben, wie seine Aufsätze aus den Jahren 1891 und 1892 zeigen (vergl. dazu aber auch ROBERT BRISART [6]). Im Vorwort (p. IX) des ersten Bandes seiner, Grundgesetze der Arithmetik، (1893) gibt er DEDEKIND Recht, daß in der Arithmetik das Gleichheitszeichen im Sinne von ,,identisch mit“" gebraucht wird und räumt ein (p. X), daß er in seiner Begriffsschrift statt vom ,Inhalt“ eines Namens besser von seiner „Bedeutung“ hätte sprechen sollen. Die „Bedeutung“ eines Namens $A$ ist für FREGE der Gegenstand, der von $A$ bezeichnet wird. Jetzt steht bei FREGE ([20], pp. 9-11)

\section{$A \equiv B$ für „A und B bezeichnen denselben Gegenstand“}

und „”“ ist nichts anderes als ein Zeichen für die Identität. (Statt $A \equiv B$ schreibt FREGE von 1893 an jedoch $A=B$; wir ziehen es vor, weiterhin das Zeichen ,„” zu verwenden). ${ }^{11}$ Es ist klar, daß weiterhin die schon oben genannten Axiome (i) \& (ii) auch hier gelten. Es ist auch klar, daß die neue Definition von der jeweils betrachteten

\footnotetext{
${ }^{10}$ FREGE sprach in seiner ,Begriffsschrift ‘ [17], p. 1, von einer Sprache der ,allgemeinen Größenlehre“, wobei zu beachten ist, daß die Mathematik für FREGE immer noch eine „Größenlehre“ war. Siehe dazu auch FREGEs Schrift ,Booles rechnende Logik und die Begriffsschrift" (etwa aus den Jahren 1880/1881), die in seinen ,Nachgelassene(n) Schriften', pp. 9-52, dort p. 13, abgedruckt ist.

${ }^{11}$ Vergleiche dazu auch eine Bemerkung von HusserL aus dem Jahre 1890, die er für den geplanten zweiten Band seiner ,Philosophie der Arithmetik` aufgeschrieben hat - siehe Band XII der Husserliana, Den Haag 1970, p. 344.
} 
Sprache unabhängig ist (vergl. Abschn. 6). Damit ist die Einführung des IdentitätsZeichens in den Logik-Kalkül geglückt.

Man beachte, daß in (ii) nicht wie bei LEIBNIZ eine einzelne Aussage (höherer Stufe) steht, in der über alle Formeln $\Phi$ quantifiziert wird, sondern eine unendliche Liste von einzelnen Formeln: für jede einzelne Formel $\Phi$ ist die in (ii) angegebene Formel ein Axiom.

Wir wollen auch noch auf den Kunstgriff FREGEs hinweisen, demzufolge im Unterschied zur LEIBNIZ'schen Definition (\#) in (ii) keine Doppelpfeile verwendet wurden. Während für LEIBNIZ die Ersetzbarkeit salva veritate eine notwendige und hinreichende Bedingung für Identität ist, ist sie hier in (ii) - übrigens wie bei ARISTOTELES in (A2) - nur eine notwendige Bedingung für Identität. In (ii) wird also nicht versucht, den Begriff der Identität zu definieren, sondern nur die Gültigkeit von einigen Eigenschaften gefordert, die zum Inhalt des Begriffes der Identität gehören. FREGE mußte auf die hinreichende Bedingung verzichten, weil ihre Gültigkeit nur unter der Voraussetzung des Individuations-Prinzips zu rechtfertigen wäre, aber dieses Prinzip in der Mathematik ungültig ist - vergl. Abschn. $6 \& 7$. Überdies wäre die hinreichende Bedingung nur in einer infinitären Sprache oder einer Sprache der 2. Stufe ausdrückbar.

Aufgrund der Abweichung von der LEIBNIZ'schen Formulierung, ist es fraglich, ob in der Liste der Axiome (i) \& (ii) alles, was über die Identität (in einer Sprache der 1. Stufe) ausgesagt werden kann, noch enthalten ist. Bei FREGE blieb diese Frage offen. Sie wurde erst im Jahre 1929, von KURT GÖDEL in seiner Dissertation ,Über die Vollständigkeit des Logikkalküls‘ [23] im positiven Sinne beantwortet. Wir werden auf den Beweis dieses GöDEL'schen Satzes im folgenden Abschn. 9 kurz eingehen.

\section{Eine implizite Definition des Begriffes der ,Identität‘ (KURT GöDEL)}

Wir wollen abschließend zeigen, daß alles, was vom Inhalt des Begriffes der Identität in Sprachen der 1. Stufe ausdrückbar ist, aus den Axiomen des FREGE'schen Systems (i) \& (ii) mit den Mitteln des Logik-Kalküls für die übrigen Konstanten $\neg, \Rightarrow, \forall$ herleitbar ist.

Der „Inhalt des Begriffes der Identität“ ist dabei die Gesamtheit aller Aussagen aus einer vorgegebenen Sprache $\mathscr{L}$ der 1 . Stufe, die in allen $\mathscr{L}$-Strukturen gelten, in denen das 2-stellige Prädikat ,„” durch die tatsächliche Identität (Einerleiheit) interpretiert wird.

Um sicherzustellen, daß im Beweis des folgenden Satzes nur solche Informationen verwendet werden, die ausdrücklich vorausgesetzt werden, wollen wir im Folgenden das Zeichen „”“ wie ein „leeres Zeichen“ verwenden, d.h. als ein Zeichen, über dessen Inhalt vorab noch gar nichts verabredet sein soll. Es soll kein „Symbol“ sein, also kein Zeichen, das bereits vorab mit irgendeinem Inhalt verknüpft ist. Es wird nur verabredet, daß es den sämtlichen Axiomen der folgenden Liste genügen soll:

(i) $\forall x(x \equiv x)$,

(ii) für jede $\mathscr{L}$-Formel $\Phi$ die Aussage: $\forall x \forall y\left(x \equiv y \Rightarrow\left(\Phi \Rightarrow \Phi^{*}\right)\right)$. 
Dabei ist $\mathscr{L}$ irgendeine formale Sprache (der 1. Stufe) mit den üblichen logischen Zeichen $\neg, \Rightarrow, \forall$ als einzigen logischen Zeichen, die das Zeichen $\equiv$ als eines der auBerlogischen 2-stelligen Relations-Zeichen enthält, und in (ii), wie bereits mehrfach gesagt wurde, $\Phi$ eine beliebige $\mathscr{L}$-Formel, in der die Variable $x$ frei vorkommt und $y$ eine Variable, die in $\Phi$ frei-einsetzbar ist, und $\Phi^{*}$ aus $\Phi$ dadurch hervorgeht, daß an einigen Stellen, an denen $x$ frei vorkommt, $x$ durch $y$ ersetzt wird. Wir können jetzt den folgenden Satz beweisen:

Satz 2 Für jede formale Sprache $\mathscr{L}$ der ersten Stufe, die das 2-stellige RelationsZeichen $\equiv$ unter ihren außerlogischen Zeichen enthält, und die mit den üblichen logischen Zeichen $\neg, \Rightarrow, \forall$ ausgestattet ist, gilt, daß jede $\mathscr{L}$-Formel $\Psi$, die in allen solchen $\mathscr{L}$-Strukturen gilt, in denen die Relation $\equiv$ durch die Identität interpretiert wird, auch aus den üblichen Axiomen für $\neg, \Rightarrow, \forall$ zusammen mit der folgenden Liste (i) \& (ii) von $\mathscr{L}$-Formeln rein syntaktisch mit den üblichen Herleitungsregeln des Prädikatenkalküls der 1. Stufe gewonnen werden kann,

(i) $\forall x(x \equiv x)$,

(ii) für jede $\mathscr{L}$-Formel $\Phi$ die Aussage: $\forall x \forall y\left(x \equiv y \Rightarrow\left(\Phi \Rightarrow \Phi^{*}\right)\right)$.

Zum Beweis des Satzes (in enger Anlehnung an GöDEL [23], pp. 116-118) nehmen wir an, es gäbe eine $\mathscr{L}$-Formel $\Psi$, die in allen $\mathscr{L}$-Strukturen gilt, in denen die Relation $\equiv$ durch die Identität interpretiert wird, die aber nicht unter Verwendung der üblichen Axiome der Prädikatenlogik der 1. Stufe für $\neg, \Rightarrow, \forall$, zusammen mit den Axiomen aus den Listen (i) \& (ii) herleitbar ist. Dann ist die Formelmenge $\{$ (i), (ii), $\neg \Psi\}$ syntaktisch widerspruchsfrei und es müßte nach dem GÖDEL'schen VollständigkeitsSatz (für den Prädikatenkalkül ohne Identität [23], pp. 104-116) eine $\mathscr{L}$-Struktur $\mathfrak{U}=\langle A, \ldots\rangle$ geben, in der (i), (ii) und $\neg \Psi$ gelten. Die Interpretation von $\equiv$ in $\mathfrak{U}$ definiert auf $A$ eine Äquivalenzrelation. Für $a \in A$ sei $[a]$ die Äquivalenzklasse von $a$, also die Menge aller mit $a$ äquivalenten Elemente von $A$. Sei $B=\{[a] ; a \in A\}$. Da (i) $\&$ (ii) in $\mathfrak{U}$ gelten, kann unter Verwendung der Projektion, die $a$ auf $[a]$ abbildet, auch $B$ ganz kanonisch zu einer $\mathscr{L}$-Struktur gemacht werden, $\mathfrak{B}=\langle B, \ldots\rangle$. Dann wird die Relation $\equiv$ in $\mathfrak{B}$ durch die Identitätsrelation interpretiert! Durch Induktion über den Formelaufbau zeigt man, daß für alle $\mathscr{L}$-Formeln $\Theta\left(x_{1}, \ldots, x_{n}\right)$ und alle $a_{1}, \ldots, a_{n} \in A, \Theta\left(a_{1}, \ldots, a_{n}\right)$ in $\mathfrak{U}$ genau dann gilt, wenn $\Theta\left(\left[a_{1}\right], \ldots,\left[a_{n}\right]\right)$ in $\mathfrak{B}$ gilt. Daraus folgt, daß auch $\mathfrak{B}=\langle B, \ldots\rangle$ ein Modell von $\neg \Psi$ ist. Das widerspricht unserer Annahme, daß $\Psi$ in allen $\mathscr{L}$-Strukturen gilt, in denen $\equiv$ durch die Identität interpretiert wird. Also muß $\Psi$ doch herleitbar sein.

Aus dem angegebenen Satz ergibt sich für die Logik der 1. Stufe eine Kennzeichnung der Identität, denn einerseits gelten alle Axiome, die in den Listen (i) und (ii) aufgeführt werden, auch für die Identitätsrelation und andererseits sind alle Eigenschaften, die die Identitätsrelation besitzt und die in einer Sprache der 1. Stufe ausdrückbar sind, aus den beiden Listen von Axiomen (i) und (ii) herleitbar. Alles, was vom Inhalt der Identität in Sprachen der 1. Stufe ausdrückbar ist, ist also aus den beiden von FREGE angegebenen Listen von Axiomen (i) und (ii) herleitbar. Dieser (auf die Ausdrückbarkeit in Sprachen der 1. Stufe begrenzte) Inhalt der Identität ist daher axiomatisierbar und kann somit - wie in (i) \& (ii) angegeben - in einer impliziten 
Definition eingefangen werden. Wir haben also das Ergebnis: Der Begriff der Identität, wenn er auf die Logik der 1. Stufe eingeschränkt wird, ist implizit definierbar, und zwar ist die in (i) \& (ii) angegebene Liste von Axiomen eine derartige implizite Definition.

Wir erkennen zugleich, daß die Identität den klassischen Grundbegriffen der Logik (,,nicht“", ,und“", ,oder“, ,für alle“,...) an die Seite gestellt werden kann, da auch ihre Bedeutung (im Unterschied zur umgangssprachlichen Gleichheit) immer eindeutig ist und sie insofern eine ,logische Konstante“ ist.

\section{Schlußbetrachtung}

Die Begriffe der Gleichheit und der Identität sind, wie wir gesehen haben, weder in der Umgangssprache noch in der Fachsprache der Mathematik synonym. Aber dennoch wird in der Mathematik immer noch dort, wo man von Identität sprechen sollte, zumeist von Gleichheit gesprochen. Das ist nicht falsch, da identische Objekte auch einander gleich sind. In dieser Ausdrucksweise wird jedoch das eigentlich Gemeinte verschleiert. Es handelt sich dabei wohl um ein Zugeständnis an den seit Jahrhunderten üblichen Sprachgebrauch.

Dieser Sprachgebrauch ist nicht ganz unbegründet. Wenn man in Anlehnung an AlchWoÁRismi (ca. 820), AdAM RiES (1518/1522), JOHANN BERNHARD BASEDOW (1774), LEOPOLD KRONECKER (1887) und manchen anderen bei der Begründung des Zahlbegriffs die Auffassung des Nominalismus zugrunde legt (so, wie es auch heute noch auf den Schulen üblich ist), dann sind die sprachlichen Darstellungen der Zahlen (etwa als Zahlwörter oder als Folgen von Ziffern im dezimalen Stellenwertsystem) die Zahlen selbst. Dann ist beispielsweise $7+5=5+7$ keine Identität, sondern „nur“ eine Gleichheit, da ja die hingeschriebenen Zeichenreihen offenbar verschieden sind.

Der nominalistischen Auffassung steht die Auffassung gegenüber, daß man es in der Mathematik doch mit Dingen zu tun hat, wobei die Natur der Dinge zwar unerheblich ist, ihre gegenseitigen Beziehungen jedoch von ausschlaggebender Bedeutung sind. Eine solche Auffassung wurde im Falle der natürlichen Zahlen bereits von Platon (,Philebos'56d-e, ,Politeia‘ 526a-b) und Euklid (,Elemente‘, Buch 7) begründet. Hier sind die natürlichen Zahlen endliche Mengen von Einheiten, also Dinge, die von ihren Namen verschieden sind (vergl. Platon, ,7. Brief ${ }^{\star}, 342 b$ ). Von DiOPHANT an bis CHRISTIAN WOLFF (1710) und ABRAHAM KÄSTNER (1766) haben fast alle Mathematiker diese platonisch-euklidische Auffassung vertreten. Auch in der seit RICHARD DEDEKIND (1888) üblich gewordenen strukturalistischen Auffassung des Zahlbegriffs (siehe Abschn. 7 \& 8) sind die Zahlwörter nur die Namen von Dingen, die im Sinne einer zugrunde gelegten Struktur als Zahlen anzusehen sind, und daher ist auch hier $7+5=5+7$ eine Identität.

Der Begriff der Identität ist erst von der Mitte des 19. Jahrhunderts an in die Mathematik (und zunächst nur in die Mathematische Logik) eingedrungen. Er war ungewohnt und noch nicht vertraut. Allmählich wurde es üblich zu betonen, daß man zwar noch die alte Ausdrucksweise verwenden wolle und von Gleichheit sprechen werde, aber eigentlich die Identität meine. 
Alfred North WhiteheAd \& BerTRAND Russell schrieben in ihren ,Principia Mathematica“ ([42], Bd. 1, 1910, p. 168), daß sie den Ausdruck ,x ist mit $y$ identisch" in der Form,$x=y$ “ schreiben werden, aber dennoch,$x=y$ “ als , sign of equality" lesen wollen.

Bei Nicolas BourbaKI heißt es in seinem Werk ,Théorie des ensembles - Fascicule de Résultats‘ [5], p. 9) ganz genauso:

„En écrivant deux symboles de part et d'autre du signe «=»(qui se lit «égale»), on a une relation dite relation d'égalité, qui signifie que ces deux symboles représentent le même élément."

[Wenn man auf beiden Seiten des Zeichens „=“ (gelesen: ,ist gleich“) jeweils ein Symbol hinschreibt, dann hat man eine Relation, die Gleichheitsrelation genannt wird und die andeutet, daß die beiden Symbole dasselbe Element darstellen.]

Diese und viele ähnlich lautende Belege zeigen, daß man in guter Gesellschaft ist, wenn man sich dafür entscheidet, einen Ausdruck wie $a=b$ als Gleichung zu lesen, aber zugleich betont, daß damit eigentlich eine Identifikation gemeint ist. Es wäre auch nicht falsch, $a=b$ als Äquivalenz zu lesen, denn wenn $a$ und $b$ identisch sind, dann sind sie auch äquivalent, aber dann wäre das eigentlich Gemeinte ziemlich verfehlt. Am einfachsten wäre es jedoch, $a=b$ als das, was es sein soll, nämlich als Identifikation zu lesen und von „=“ als dem Zeichen der Identität zu sprechen.

Funding Note Open Access funding enabled and organized by Projekt DEAL.

Hinweis des Verlags Der Verlag bleibt in Hinblick auf geografische Zuordnungen und Gebietsbezeichnungen in veröffentlichten Karten und Institutsadressen neutral.

Open Access This article is licensed under a Creative Commons Attribution 4.0 International License, which permits use, sharing, adaptation, distribution and reproduction in any medium or format, as long as you give appropriate credit to the original author(s) and the source, provide a link to the Creative Commons licence, and indicate if changes were made. The images or other third party material in this article are included in the article's Creative Commons licence, unless indicated otherwise in a credit line to the material. If material is not included in the article's Creative Commons licence and your intended use is not permitted by statutory regulation or exceeds the permitted use, you will need to obtain permission directly from the copyright holder. To view a copy of this licence, visit http://creativecommons.org/licenses/by/4.0/.

\section{Literatur}

1. Angelelli, I.: Friends and opponents of the substitutivity of identicals in the history of logic. In: von Schirn, M. (Hrsg.) Studien zu Frege, Band 2: Logik und Sprachphilosophie, Stuttgart, S. 141166 (1976)

2. Archimedes: Opera Omnia, Vier Bände, herausgegeben von J.L. Heiberg (Bände 1-3, Leipzig 18811915) und Dold-Samplonius, Y., Hermelink, H., Schramm, M. (Bd. 4, Stuttgart, 1975). Eine deutsche Übersetzung hat Czwalina, A. bei der Wissenschaftliche Buchgesellschaft, Darmstadt 1972 herausgegeben

3. Arnauld, A.: La Logique, ou L'Art de penser. Paris (1662)

4. Boole, G.: An Investigation of the Laws of Thought. London (1854)

5. Bourbaki, N.: Théorie des ensembles, Fascicule de résultats. Éléments de Mathématique, Bd. 1. Paris, Hermann (1939), 3 Aufl., 1958 
6. Brisart, R.: Le problème de l'abstraction en Mathématiques, l'écart initial de Husserl, par rapport à Frege entre 1891 et 1894. In: von Brisart, R. (Hrsg.) Husserl et Frege, les ambiguïtés de l'antipsychologisme, Paris, S. 13-47 (2002)

7. Chang, C.-C., Keisler, H.J.: Model Theory. North-Holland, Amsterdam (1973)

8. Couturat, L.: In: Opuscules et Fragments inédits de Leibniz, Paris (1903). Nachdruck: Olms-Verlag, Hildesheim (1961)

9. Couturat, L.: Les Principes des Mathématiques. Alcan, Paris (1905)

10. Dedekind, R.: Was sind und was sollen die Zahlen? Vieweg, Braunschweig (1888)

11. Dilworth, C.: Identity, equality and equivalence. Dialectica 42, 83-92 (1988)

12. Diogenes Laërtios: Leben und Meinungen berühmter Philosophen. Aus dem Griechischen übersetzt von O. Apelt et al. Meiner, Hamburg (1967)

13. Euklid: In: Heiberg, J.L., Menge, H. (Hrsg.) Opera gr. et lat, Leipzig, 1883-1916. Eine deutsche Übersetzung der ,Elemente', Buch I-XIII, übersetzt von Clemens Thaer, erschien in der Wiss. Buchgesellschaft, Darmstadt (1962)

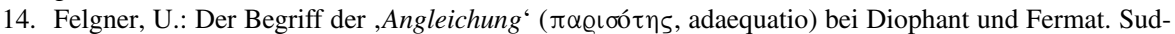
hoffs Archiv 100, 83-109 (2016)

15. Felgner, U.: Philosophie der Mathematik, in der Antike und in der Neuzeit. Birkhäuser, Basel (2020)

16. Fraenkel, A.A., Bar-Hillel, Y., Levy, A.: Foundations of Set Theory. North-Holland, Amsterdam (1973)

17. Frege, G.: Begriffsschrift, eine der arithmetischen nachgebildete Formelsprache des reinen Denkens. Nebert, Halle (1879), a/S

18. Frege, G.: Grundlagen der Arithmetik. Wilhelm Koebner, Breslau (1884)

19. Frege, G.: Über Sinn und Bedeutung, Zeitschrift für Philosophie und philosophische Kritik, Bd. 100, S. 25-50 (1892). Nachdruck in Frege: ,Kleine Schriften', Hildesheim (1967), pp. 143-162

20. Frege, G.: Grundgesetze der Arithmetik, begriffsschriftlich abgeleitet, Bd. 1, Jena (1893), Bd. 2, Jena (1903). Verlag H. Pohle. Nachdruck 1962 bei der Wissenschaftlichen Buchgesellschaft Darmstadt. Eine neue englische Übersetzung haben $\mathrm{Ph}$. Ebert \& Marcus Rossberg herausgegeben. Oxford University Press (2013). Einen Appendix „On Frege’s Logic“ schrieb Roy T. Cook

21. Frege, G.: In: von Gabriel, G., et al. (Hrsg.) Wissenschaftlicher Briefwechsel. Meiner, Hamburg (1976)

22. von Fritz, K.: Gleichheit, Kongruenz und Ähnlichkeit in der antiken Mathematik bis auf Euklid. In: von Fritz, K. (Hrsg.) Grundprobleme der Geschichte der antiken Wissenschaft, S. 430-508. De Gruyter, Berlin (1971)

23. Gödel, K.: In: Die Vollständigkeit der Axiome des logischen Funktionen-kalküls. Monatshefte für Math. u. Physik, Bd. 37, S. 349-360 (1930). Nachdruck zusammen mit der Gödel'schen Dissertation (Univ. Wien, 1929) in Band 1 der von S. Feferman et al. herausgegebenen ,Collected Works', pp. 60-100 \& 102-122, Oxford (1986).

24. Hessenberg, G.: Grundlagen der Geometrie, (posthum) herausgegeben von Dr. W. Schwan, Göschens Lehrbücherei, Gruppe I, Bd. 17, Berlin (1930). Eine erheblich erweiterte 2. Auflage gab Justus Diller heraus, Berlin (1967)

25. Hilbert, D.: Über die Grundlagen der Logik und der Arithmetik. In: Verhandlungen des 3 Internat. Mathematiker-Kongresses, Heidelberg (1904)

26. Hilbert, D., Ackermann, W.: Grundzüge der theoretischen Logik. Springer, Berlin (1928)

27. Kauppi, R.: Einige Bemerkungen zum Principium Identitatis Indiscernibilium bei Leibniz. Zeitschrift für philosophische Forschung 20, 497-506 (1966)

28. Lambert, J.H.: Anlage zur Architectonic oder Theorie des Einfachen und des Ersten in der philosophischen und mathematischen Erkenntnis, Riga (1771). Nachdruck in Lamberts Philosophischen Schriften, Bd. III, Olms Verlag (1965)

29. Leibniz, G.W.: Leibnizens mathematische Schriften, herausgegeben von C.I. Gerhardt, Sieben Bände, Bände I \& II. Asher, Berlin (1849-1850). Bände III-VII, Halle 1855-1863. Nachdruck: Olms-Verlag Hildesheim 1962

30. Leibniz, G.W.: Neue Abhandlungen über den menschlichen Verstand. Meiner Verlag, Hamburg (1971). Übersetzt von E. Cassirer

31. Leibniz, G.W.: Dialog zur Einführung in die Arithmetik u. Algebra. Frommann-Holzboog, Stuttgart (1976), übersetzt und kommentiert von E. Knobloch

32. Leibniz, G.W.: Schriften zur Logik und zur philosophischen Grundlegung von Mathematik und Naturwissenschaft. Philosophischen Schriften, Bd. IV. Wissenschaftliche Buchgesellschaft, Darmstadt (1992), herausgegeben und übersetzt von H. Herring 
33. Moore, G.E.: Identity, Proceedings Aristotelian Soc. 1, 103-127 (1900)

34. De Morgan, A.: Formal Logic, or the calculus of inference necessary and probable. Taylor and Walton, London (1847)

35. Natorp, P.: Die logischen Grundlagen der exakten Wissenschaften. Teubner, Leipzig (1910)

36. Peano, G.: Notations de Logique Mathématique - Introduction au Formulaire de Mathématique, Turin (1894). Nachdruck in Opere Scelte, Band 2, Rom 1958, S. 123-176

37. Quine, W.V.O.: Identity, ostension and hypostasis. Journal of Philosophy 47, 621-633 (1950) Nachdruck in Quine: From a Logical Point of View, Harvard Univ. Press Cambridge/Mass., S. 65-79 (1964).

38. Ramsey, F.P.: The foundations of mathematics. Proc. London Math. Soc. (2) 25, 338-384 (1926). Eine deutsche Übersetzung findet sich in F.P. Ramsey: Grundlagen, Abhandlungen zur Philosophie, Logik, Mathematik etc., S. 131-177. Frommann-Verlag, Stuttgart (1980).

39. Sullivan, P.M.: Frege's Logic. In: Gabbay, D.M., Woods, J. (Hrsg.) Handbook of the History of Logic, Bd. 3, S. 659-750. Elsevier, Amsterdam (2004)

40. Voltaire: Euvres Complètes, in 70 Bänden. Kehl (1783-1789)

41. Weyl, H.: Philosophie der Mathematik und Naturwissenschaf. Oldenbourg, München (1928). Eine dritte, wesentlich erweiterte Auflage erschien 1966 ebenfalls in München.

42. Whitehead, A.N., Russell, B.: Principia Mathematica, Bd. I. Cambridge University Press, Cambridge (1910)

43. Wittgenstein, L.: Vorlesungen 1930-1935. Suhrkamp, Frankfurt am Main (1984)

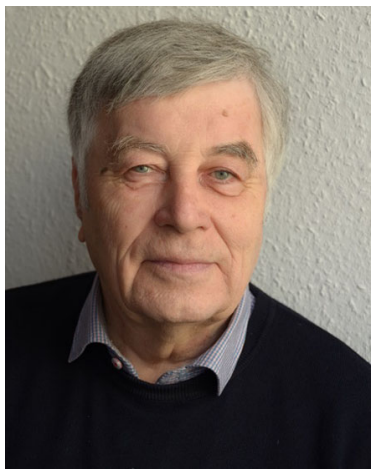

Ulrich Felgner geb. 1941, ist Professor (i.R.) für Mathematik an der Universität Tübingen. Er studierte an den Universitäten Gießen, Besançon und Frankfurt/M., promovierte 1968 in Tübingen und habilitierte sich 1973 in Heidelberg. Er wirkte als Professor an den Universitäten Heidelberg, Freiburg und Tübingen. Seine Hauptarbeitsgebiete sind Algebra, Mathematische Logik, Mengenlehre, sowie Grundlagen, Geschichte und Philosophie der Mathematik. 